\title{
Identification of a new isoform of the murine Sh2dla gene and its functional implications
}

\author{
WU LongYan, LU PeiWen, MA WeiWei, CHU CoCo, XU HePing \& QI Hai* \\ Tsinghua-Peking Center for Life Sciences, Laboratory of Dynamic Immunobiology, School of Medicine, School of Life Sciences, \\ Tsinghua University, Beijing 100084, China
}

Received October 15, 2013; accepted November 18, 2013; published online December 23, 2013

\begin{abstract}
Signaling lymphocytic activation molecule (SLAM)-associated protein (SAP) is a Src homology (SH) domain 2-containing intracellular adaptor protein that is predominantly expressed in the hematopoietic system by Tymphocytes and NK cells. SAP protein is encoded by the SH2DIA gene located on the X chromosome. Loss-of-function mutations in SAP cause the X-linked lymphoproliferative disease (XLP), a severe immunodeficiency characterized by heightened susceptibility to Epstein-Barr virus and impaired humoral immunity. Normal individuals express several functional and non-functional isoforms of SAP as a result of alternative splicing. In this study, we identify a cryptic exon in the murine Sh2dla gene. At the mRNA level, the new isoform of SAP (SAP-2) that includes this new exon is widely expressed in lymphoid tissues by C57BL/6 and 129 strains of inbred mice. SAP-2 accounts for approximately $1 \%-3 \%$ of total SAP transcripts, and it is dynamically regulated during lymphocyte activation. At the protein level, the SAP-2 isoform is a 144 amino-acid protein. Compared to the dominant 126 amino-acid SAP-1 isoform, the additional 18 amino acids are inserted into a structural region that is critical for phosphotyrosine binding. Our functional analysis in vitro indicates that SAP-2 is a non-functional isoform due to decreased protein stability. Thus, both human and mouse have multiple SAP splice isoforms that may or may not function. Modulation of relative proportions of these isoforms is potentially a mechanism whereby cells can regulate SAP-mediated biological activities.
\end{abstract}

Sh2d1a, SAP, X-linked lymphoproliferative disease (XLP), isoform

Citation: Wu LY, Lu PW, MA WW, Chu CC, Xu HP, Qi H. Identification of a new isoform of the murine Sh2dla gene and its functional implications. Sci China Life Sci, 2014, 57: 81-87, doi: 10.1007/s11427-013-4584-z

The X-linked lymphoproliferative disease (XLP), initially identified by Purtilo in 1975 [1], is a rare inherited immunodeficiency that is characterized by severe immune dysregulation after viral infection, especially by the Epstein-Barr virus [2], and abnormalities in serum IgG levels [3]. The XLP gene was initially determined to be at chromosome Xq25 by linkage analysis [4,5] and later cloned as SH2DIA [6,7]. SH2DIA encodes an SH2 domain (Src homology domain 2) containing adaptor protein that binds to immunoreceptor tyrosine-based switch motifs (ITSMs) located in the cytoplasmic tails of SLAM family receptors,

*Corresponding author (email: qihai@biomed.tsinghua.edu.cn) including SLAM (CD150, SLAMF1), CD48 (SLAMF2), 2B4 (CD244, SLAMF4), Ly9 (CD229, SLAMF3), CD84 (SLAMF5), and NK-T-B-antigen (NTB-A; SF2000 in human or Ly108 in mouse, SLAMF6) [8]. SAP knockout mice recapitulate many characteristics of the XLP syndrome, and a striking phenotype is the lack of germinal centers [9]. This is due to the fact that SAP-deficient T cells cannot stably interact with cognate B cells and deliver sufficient help to support germinal center formation [10]. Recent evidence indicates that this latter function of SAP is mainly related to Ly108 and CD84 but not to other members of the SLAM family [11,12].

The human SH2D1A gene consists of four exons, is tran- 
scribed as 2530-bp of mRNA with an open reading frame (ORF) of $462 \mathrm{bp}$, and encodes a protein of 128 amino acids $[6,13]$. Two splice isoforms have been found in human, one full-length and one shorter by 3 amino acids at the $\mathrm{C}$ terminus due to an alternative in-frame splice site. Mouse Sh2dla gene also contains four known exons, while no splice isoforms have been reported. In the present study, we have identified a new exon in the mouse Sh2dla gene that produces a novel splice isoform.

\section{Materials and methods}

\subsection{Mice}

B6 (Jax 664) and OVA $323-339$-specific TCR transgenic OT-2 (Jax 4194) mice were from the Jackson Laboratory. SAP deficient mice were kindly provided by Dr. Pamela Schwartzberg at NIH [14]. All mice were maintained under specific pathogen-free conditions, and used in accordance with governmental and institutional guidelines for animal welfare.

\subsection{Cloning and expression of SAP isoforms}

Total thymic RNA was isolated from C57BL/6 mice (6-8 weeks) by the Trizol reagent (Invitrogen, USA). cDNA was made using ReverseAid First strand synthesis kit (Thermo scientific, USA). To amplify the full coding sequence of SAP, we used the following primers: SAP-F (5'- ATGGATGCAGTGACTGTGTACC-3') and SAP-R (5'-TCATGGTGCATTCAGGCAGATATC-3'). Amplified fragments were subcloned using restriction enzymes, and inserts from several clones were sequenced. DNA and protein alignments were performed using SnapGene and DNASTAR. To express SAP in various cells, we cloned its isoforms or its direct fusions with GFP by standard molecular techniques into a custom-made MSCV-RFP vector, in which gene insert is driven by the LTR promoter while RFP is driven by an independent human ubiquitin promoter.

\subsection{Tissue and cell preparation for SAP expression analysis}

Lymph node, thymus and spleen tissues were snap-frozen in liquid nitrogen and pestled to powder before total RNA was extracted using the Trizol reagent (Invitrogen, USA). CD4 ${ }^{+}$ and $\mathrm{CD}^{+} \mathrm{T}$ cells were isolated with Microbeads by positive-selection (Miltenyi Biotec, Germany), and then stimulated with plate-bound anti-CD3 and anti-CD28 mAbs. Cells were harvested at 0,2 and $24 \mathrm{~h}$ after stimulation. $\mathrm{CD} 4^{+} \mathrm{CD}^{-}, \mathrm{CD}^{-} \mathrm{CD}^{+}, \mathrm{CD}^{-} \mathrm{CD}^{-}$, and $\mathrm{CD} 4^{+} \mathrm{CD} 8^{+}$thymocytes were sorted by standard flow cytometry using an Aria III sorter (BD Biosciences, USA). Total RNA from these cells was extracted by QIAGEN Rneasy Mini kit (QIAGEN, Germany), and cDNA was made with the Re-
verseAid First strand synthesis kit (Thermo scientific, USA). Equal amounts of total RNAs were used for reverse transcription in all samples of the same experiment.

\subsection{Real-time PCR}

Expression levels of total SAP and the SAP-2 isoform were measured by real-time PCR using SYBR premix Ex Taq reagent (TAKARA, Japan) and Applied Biosystems 7500 (ABI, USA) StepOnePlus ${ }^{\mathrm{TM}}$ Real-Time PCR System. Equal amounts of cDNA (20-40 ng) from different samples were used for each reaction of 40 amplification cycles. Data were analyzed by the $2^{-\Delta \Delta C_{\mathrm{T}}}$ method [15]. GAPDH was used as the housekeeping internal control for all experiments. The specificity of the PCR products was verified by melting curve analysis and agarose gel electrophoresis. The following primers were used: SAP-total-F (5'-TGTCCCAGACAGAAACAGGTTC-3') and SAP-total-R (5'-GCTTCTGAAACGCTGAGATGAG-3'); SAP-2-F (5'-CCAGCACTATGGATTAGCCTATG-3') and SAP-2-R (5'- TCTCGGCACTCCAAGAACC-3'); GAPDH-F (5'-TGTTCCTACCCCCAATGTGTC-3') and GAPDH-R (5'-TAGCCCAAGATGCCCTTCAGT-3').

\subsection{Transfection, retroviral production, and $T$ cell in- fection}

X-tremeGENE HP DNA Transfection Reagent (Roche, Switzerland) was used to transfect 293T or Plate-E packaging cells with indicated MSCV constructs following the manufacturer's instructions, as previously described [16]. To infect T cells, we spun $3 \times 10^{5}$ activated T cells at $1500 \times g$ with viral supernatants in the presence of $1 \mu \mathrm{g} \mathrm{mL}$ polybrene (Sigma, USA) and $10 \mathrm{ng} \mathrm{mL}{ }^{-1} \mathrm{IL}-2$ (Peprotech, USA) for $2 \mathrm{~h}$ at $32^{\circ} \mathrm{C}$. Infected $\mathrm{T}$ cells were then transferred into new wells with fresh media supplemented with $10 \mathrm{ng}$ $\mathrm{mL}^{-1}$ IL-2.

\subsection{Detection of intracellular SAP protein expression}

Cells were fixed with $1 \%$ paraformaldehyde, permeablized with $0.1 \%$ saponin, and stained with an AlexaFluor647conjugated monoclonal anti-SAP antibody (a kind gift from Dr. Andre Veillette, Clinical Research Institute of Montreal) [17].

\subsection{T-B cell conjugation assay}

A previously described protocol was used with some modifications [10]. $\mathrm{CD}^{+} \mathrm{T}$ cells were isolated from SAPdeficient OT-II TCR transgenic mice, activated with platebound anti-CD3/CD28 mAbs (BioXcell, USA), and then infected with MSCV-SAP-1-RFP, MSCV-SAP-2-RFP or control MSCV-RFP virus. Splenic B cells were stimulated with LPS (Sigma, USA) for $3 \mathrm{~d}$. T cells and B cells were 
stained with $\mathrm{CMF}_{2} \mathrm{HC}$ and DDAO (Invitrogen, USA), respectively. B cells were pulsed with $\mathrm{OVA}_{323-339}$ peptide (Genscript, USA) at a final concentration of $0,0.1$, and 0.5 $\mu \mathrm{mol} \mathrm{L} \mathrm{L}^{-1}$ for $30 \mathrm{~min}$ before being mixed with the $\mathrm{T}$ cells at a ratio of $2: 1$. The $\mathrm{T}$ and $\mathrm{B}$ cells were then spun together at $300 \times g$ and incubated for $30 \mathrm{~min}$. After being vortexed for $40 \mathrm{~s}$, the cell mixture was analyzed on an LSR II cytometer (BD Biosciences, USA). The percentages of $\mathrm{CMF}_{2} \mathrm{HC}^{+}$$\mathrm{DDAO}^{+}$doublets within $\mathrm{RFP}^{+}$(infected) and $\mathrm{RFP}^{-}$(uninfected) cells in different groups were quantitated as respective T-B cell conjugation rates. Data was analyzed with FlowJo (Tree Star, USA) and GraphPad Prism (GraphPad Software, Inc., USA).

\section{Results and discussion}

During our routine cloning of the SAP coding sequence by RT-PCR using cDNA prepared from B6 thymocytes, we noticed that one clone was larger than the other three picked clones, as evidenced by electrophoresis on the agarose gel (Figure 1A, clone \#2). Sequencing analyses showed that while all the other three clones contain the correct 381-bp SAP coding sequence as in the NCBI reference sequence (NM_011364.3) for Sh2dla, the clone \#2 sequence was 435 bp long (GenBank accession No. KF811030). As shown in Figure 1B, an additional 54-bp sequence was inserted between the first $137 \mathrm{bp}$ and the last $244 \mathrm{bp}$ of the canonical SAP coding sequence. Because the coding sequence contained in exon 1 of the Sh2dla gene is precisely 137 bp long, these data suggest that the clone \#2 cDNA is derived from an alternatively spliced SAP mRNA species, and there should be a 54-bp cryptic exon in the Sh2dla gene.

We searched in the first intron of the Sh2dla gene for this putative exon. As shown in Figure 2A, the first intron of Sh2dla is 12427 bp in length according to NCBI mouse genome Build 37.2, and a perfect match of the 54-bp DNA sequence is found between nucleotide 4866 and 4919 of this intron. The non-coding sequence immediately $5^{\prime}$ to this 54-bp segment ends with an AG dinucleotide, conforming to a regular splice acceptor site characteristic of a U2-type intron, while the sequence immediately $3^{\prime}$ to the 54-bp segment starts with a GT dinucleotide, conforming to a regular splice donor site (Figure 2A). A "TTGAC" sequence is found 27-bp upstream of the 54-bp segment, conforming to a usual branch site $(\mathrm{C} / \mathrm{T}) \mathrm{T}(\mathrm{A} / \mathrm{G}) \mathrm{A}(\mathrm{C} / \mathrm{T})$ for splicing [18]. These analyses indicate that the 54-bp segment is a bona fide exon. We thus term this segment as exon2' to differentiate it from the canonical exon2, and term the 5-exon SAP product isoform 2 (murine SAP-2, Figure 2B), while using isoform 1 (murine SAP-1) to denote the original 4-exon SAP product. Inclusion of this 54-bp exon2' between exon 1 and 2 in the SAP-2 isoform does not alter the original reading frame and is predicted to produce a protein of 144 amino acids (Figure 2C). On the other hand, inclusion of exon2' in place of exon2, also a possible splicing product, will lead to a frame shift and generate a premature stop codon that is predicted to terminate the translation of 13 amino acids downstream of exon2' (Figure 2D).

Alternatively spliced isoforms may serve different biological functions as different proteins. The SAP-1 isoform has so far been the only known isoform and assumed to be responsible for all functions of SAP. The two available SAP-deficient mouse strains do not differentiate the two isoforms, because in one strain the exon 1 is deleted [19] while the exon3 is targeted in the other [14]. Previously used PCR primers for RT-PCR analysis cannot differentiate these two isoforms.

To begin to differentiate SAP-1 and SAP-2 expression and function, we designed isoform-specific primers. As shown in Figure $3 \mathrm{~A}$, the primer set- 1 detects both SAP-1 and SAP-2 isoforms, while primer set-2 specifically detects the SAP-2 isoform. Amplicons from both sets of primers span exon-intron junctions and thereby minimize interference of mRNA quantitation by genomic DNA contamination. No suitable primers can be designed to specifically amplify the SAP-1 but not the SAP-2 isoform. We then investigated the expression pattern of SAP-2 in adult mouse lymphoid tissues. As shown in Figure 3B, similar to total SAP expression, SAP-2 was most highly expressed in the thymus as compared to the spleen or the lymph node. On the other hand, as a percentage of total SAP expression, the

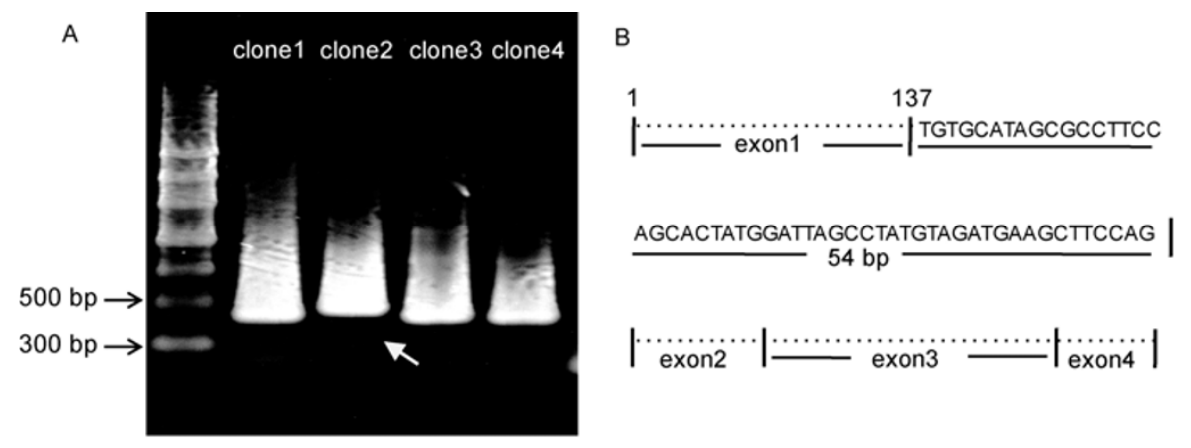

Figure 1 Cloning of SAP by PCR. A, SAP coding sequence was amplified from cDNA made from B6 thymic total RNA. Four clones were analyzed by electrophoresis on the agarose gel. B, The sequence of the clone \#2. Sequences denoted by exon1, 2, 3, and 4 were identical to NCBI reference sequence for SAP and omitted here for clarity. 

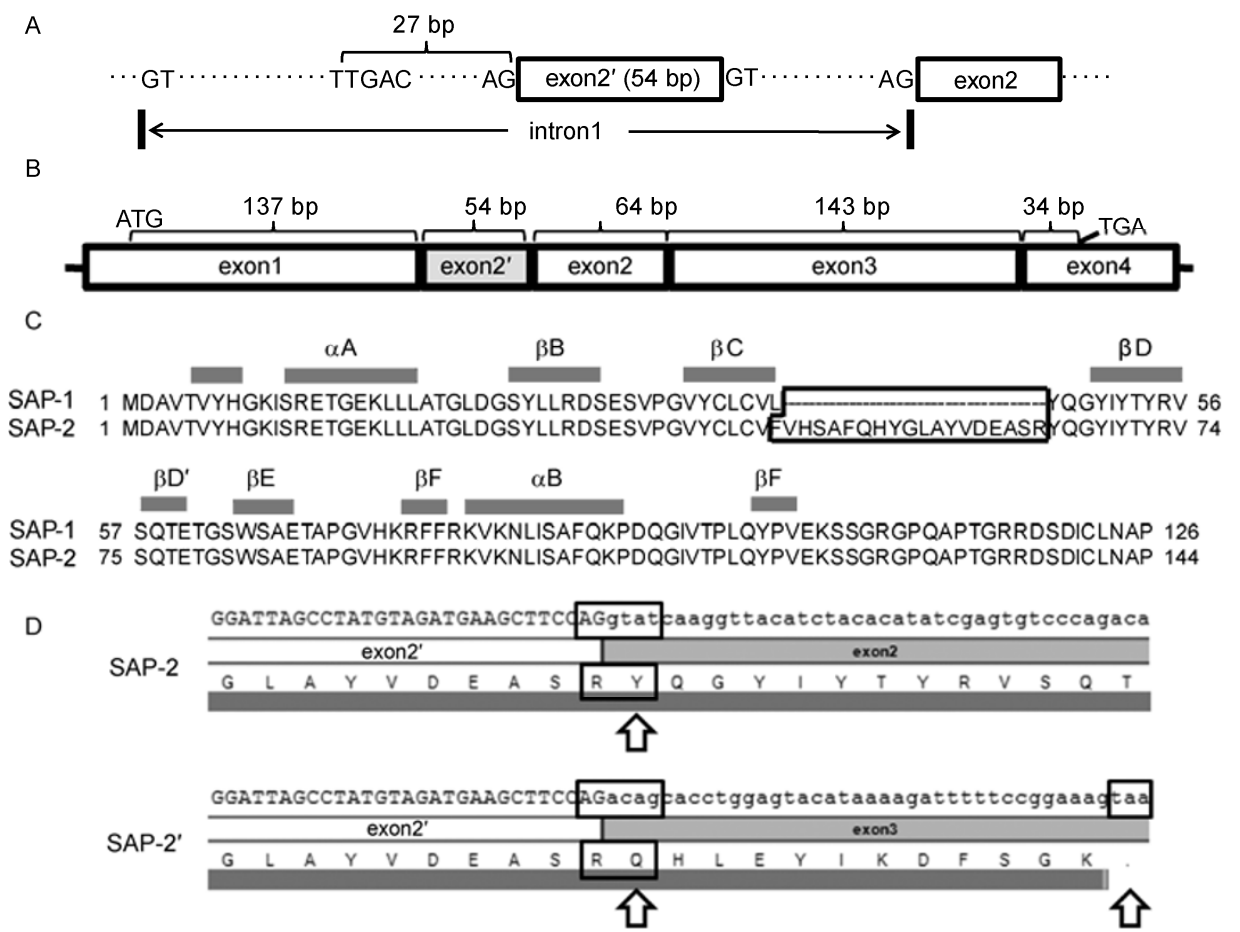

Figure 2 Sequence analysis of the SAP-2 isoform. A, Location of the cryptic exon2' in the context of intron 1 . Nucleotides corresponding to putative splicing acceptor, donor and branch site were identified. B, Schematic presentation of the 5-exon configuration of the SAP-2 isoform. C, Alignment of deduced SAP-1 and SAP-2 protein sequences. Secondary structures of SAP-1 are labeled according to the study by Morra et al. [23]. D, Two ways of alternative splicing of exon2' in relationship to exon2. Direct ligation of exon2' and exon3 with exon2 skipped is predicted to result in a frame shift and premature translational termination.

highest level of SAP-2 was seen in the lymph node (Figure 3C). SAP-2 expression was not a peculiar phenomenon of the C57BL/6 strain, as 129 mice similarly expressed the SAP-2 isoform in the thymus, spleen, and lymph node, except that the highest level of SAP-2 was seen in the spleen (Figure 3D). In thymus, SAP-2 and the total SAP exhibited a similar pattern of expression among the single-positive cells, double-positive cells and double-negative cells (Figure $3 \mathrm{E}$ ). Upon in vitro activation with anti-CD3/28 mAbs, SAP-1 expression in both $\mathrm{CD}^{+} \mathrm{T}$ and $\mathrm{CD}^{+} \mathrm{T}$ cells was decreased, as previously reported [20]. In contrast, SAP-2 expression was increased in $\mathrm{CD}^{+} \mathrm{T}$ and particularly $\mathrm{CD}^{+}$ $\mathrm{T}$ cells $24 \mathrm{~h}$ following activation (Figure $3 \mathrm{~F}$ and $\mathrm{G}$ ). Taken together, these data indicate that, at the level of whole tissues, SAP-2 is a minor but constitutively expressed isoform accounting for approximately $1 \%-3 \%$ of total SAP mRNA. Its expression is likely regulated in kinetics different from that of SAP-1.

SAP-1 is an SH2-domain containing adaptor protein, and its functions depend on SH2-mediated interactions with phospotyrosine (pY)-containing peptides such as those present in the cytoplasmic domain of SLAM family proteins $[7,21]$. Based on the crystal structure of SAP SH2 domain $[22,23]$, the additional stretch of 18 amino acids coded by exon 2 ' is inserted immediately before the $\beta \mathrm{D}$ strand (Figure $2 \mathrm{D})$. Almost all residues of the $\beta \mathrm{D}$ strand are important for the SAP interactions with the phosphotyrosine (pY)-containing ITSM motif. It seems that the compact architecture of an SH2 domain would not be able to accommodate such drastic addition without affecting its ability to bind to an ITSM motif. On the other hand, if the 18 amino acids can be arranged in a flexible loop configuration, they could in principle create docking sites for interactions with other signaling molecules. To test functional activities of SAP-2, we used an in vitro T-B cell conjugation assay [10]. It is well established that SAP-deficient T cells cannot form stable adhesion to antigen-presenting $B$ cells, and that acute complementation of SAP-1 protein by retroviral transduction can rescue this deficiency. Such rescue critically depends on the ability of SAP-1 to bind to the ITSM motif [10]. In fact, our systematic mutagenesis analyses indicated that no residues of SAP-1 other than those involved in ITSM binding were required for such rescue (unpublished data). Therefore, we compared SAP-1 and SAP-2 for their abilities to rescue SAP-deficient T cells. As shown in Figure 4, while transduction of SAP-1 in $\mathrm{Sap}^{-1-} \mathrm{T}$ cells improved their conjugate formation with antigen-presenting B cells, SAP-2 complementation failed to do so. These data indicate that SAP-2 either cannot bind to ITSM or it is simply an unstable protein. To further differentiate these two possibilities, we stained these cells for intracellular SAP protein using a monoclonal anti-SAP antibody [17]. As shown in Figure 5A, only MSCV-SAP-1-RFP but not MSCV-SAP-2- 


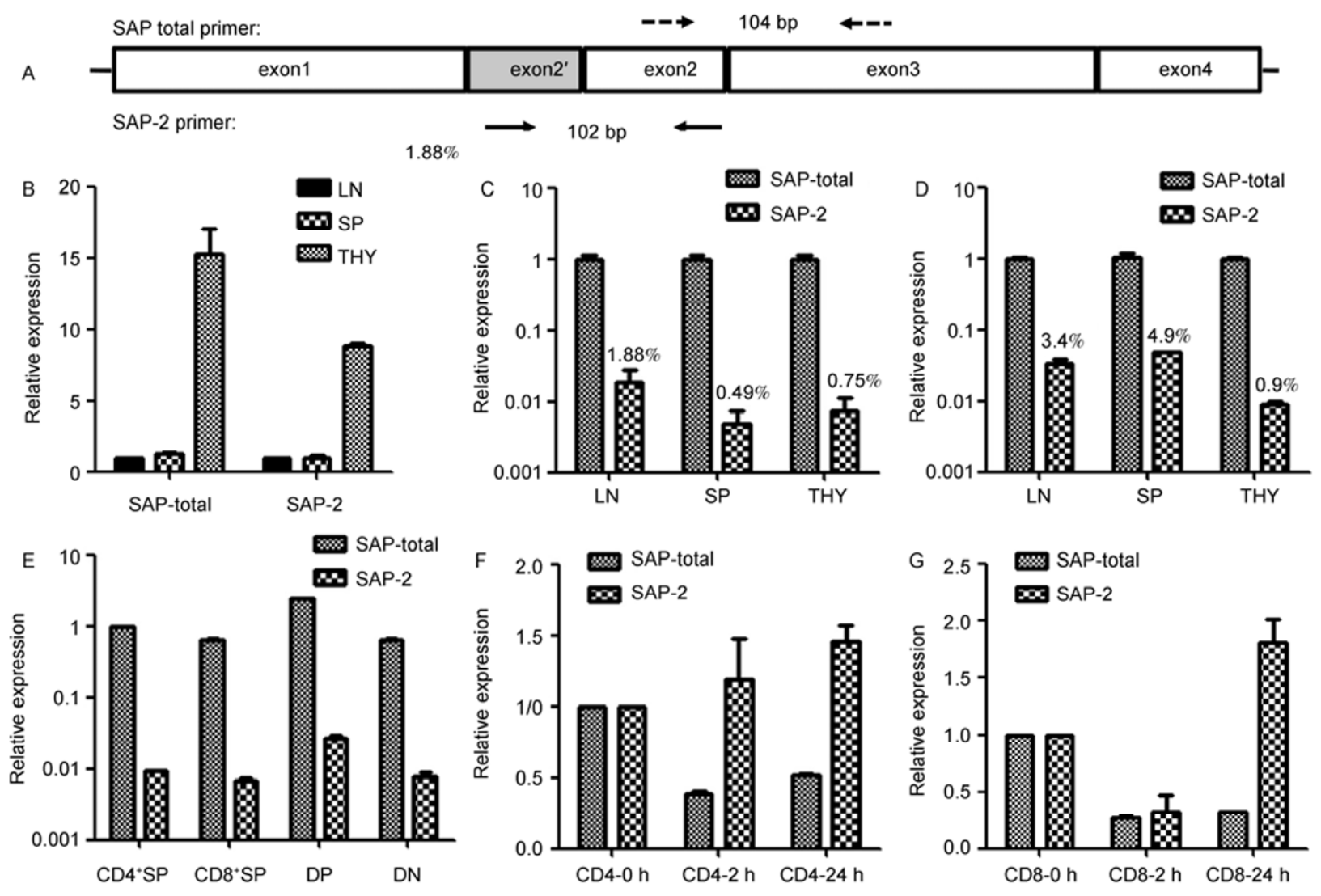

Figure 3 The expression pattern of the SAP-2 isoform. A, Design of the isoform 2-specific primers. Note amplicons from SAP-total and SAP-2 primers were designed to be adjacent on mature mRNA and of essentially the same size in order to allow fair quantitative comparison by real-time PCR. B, The mRNA expression levels of total SAP and SAP-2 in thymus (THY), spleen (SP) and lymph nodes (LN). After normalization to respective internal GAPDH control, the level in the LN group was set to be an arbitrary unit 1. Relative expression levels of SAP-2 as a proportion of the total SAP in the thymus, spleen and lymph nodes of C57BL/6 mice (C) or 129 mice (D). E, Total SAP and SAP-2 expression in different populations of thymocytes. SP, single positive; DP, double positive; DN, double negative. Total SAP and SAP-2 expression in CD4 (F) and CD8 T cells (G) at indicated time points following in vitro activation. All data are shown as mean \pm SEM and represent 2 to 4 independent experiments.
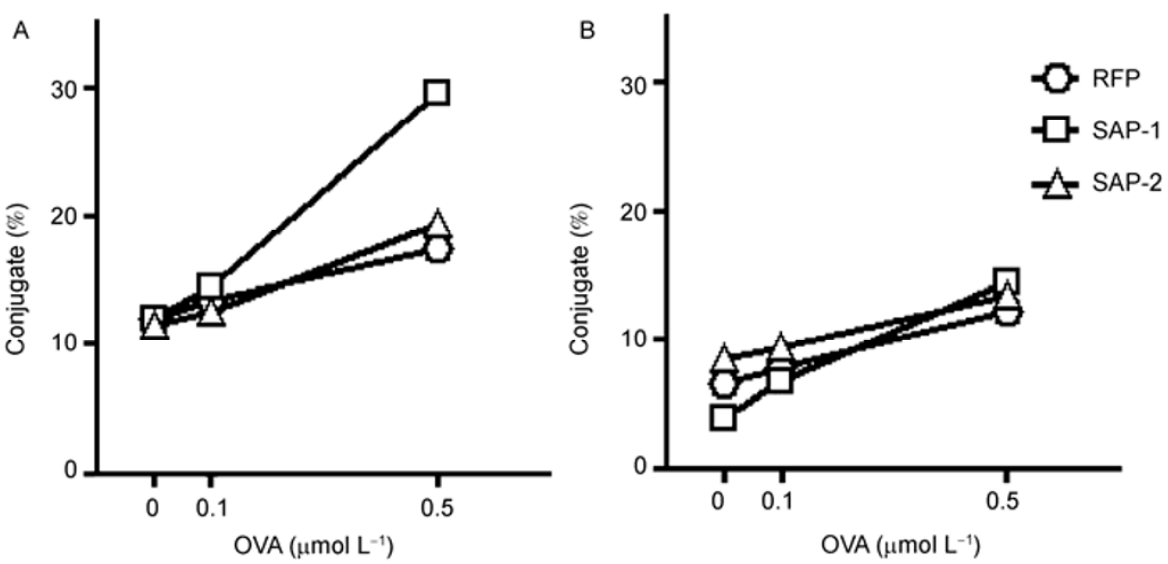

Figure 4 SAP-2 fails to rescue SAP-deficient $\mathrm{T}$ cells for optimal T-B cell conjugation. SAP ${ }^{-/}$OT-II $\mathrm{T}$ cells were infected with MSCV-RFP, MSCV-SAP-1-RFP or MSCV-SAP-2-RFP and tested in conjugation assay. Plotted are conjugate frequencies of infected (RFP ${ }^{+}$gated, left panel) and (RFP ${ }^{-}$ gated, right panel). One of three separate experiments with similar results is shown.

RFP group expressed detectable levels of intracellular SAP protein, suggesting that SAP-2 might not be stable. An alternative possibility is that SAP-2 might still be normally expressed and stable, but the epitope recognized by the monoclonal antibody is altered so that SAP-2 becomes undetectable. To test this, we expressed fusion protein between GFP and SAP-1 or SAP-2 or GFP alone in 293T cells by the MSCV-RFP vector. Because two independent pro- 

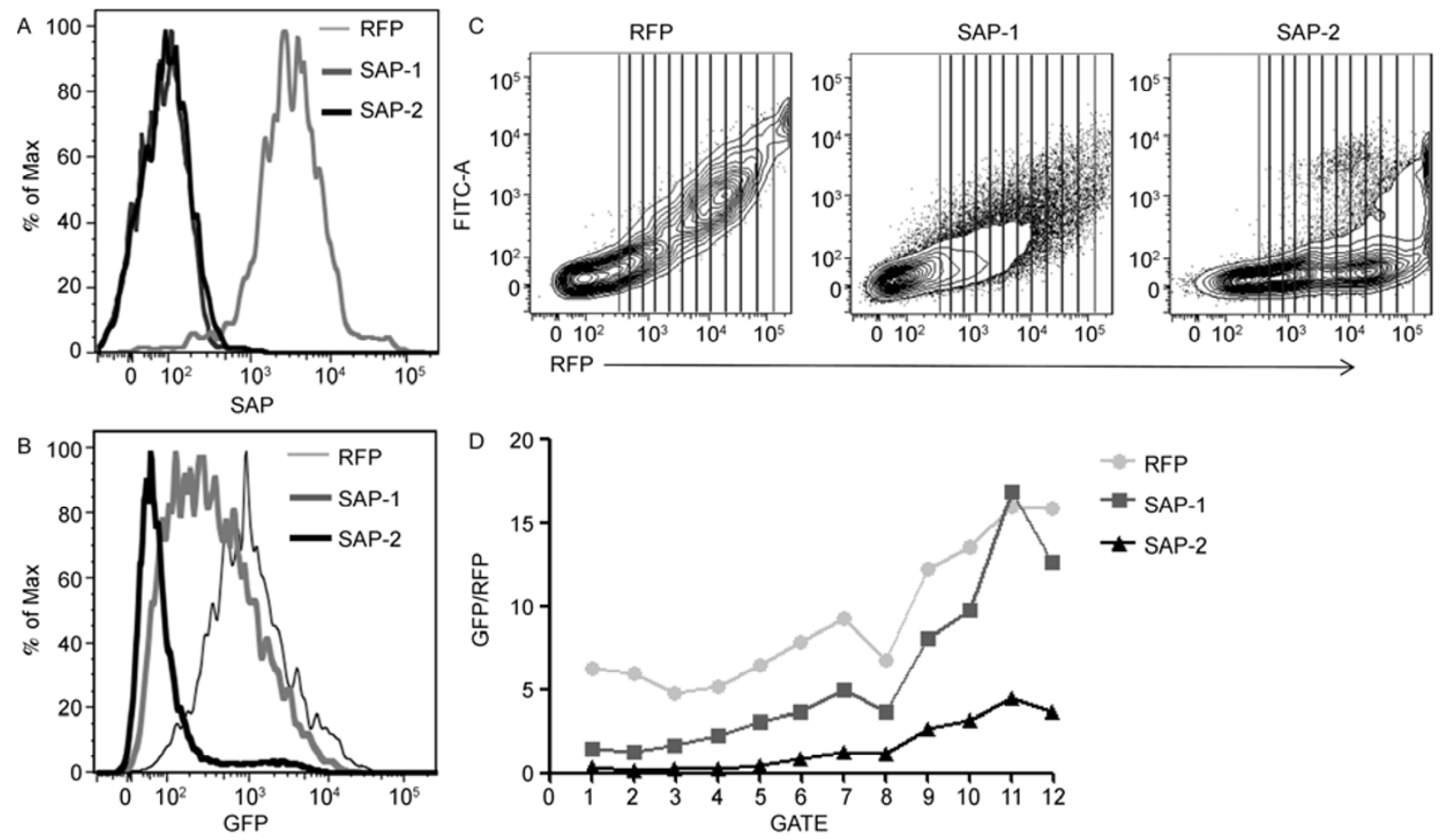

Figure 5 SAP-2 is unstable. A, SAP protein levels in T cells transfected with MSCV-RFP, MSCV-SAP-1-RFP or MSCV-SAP-2-RFP, as detected by intracellular staining with an anti-SAP antibody (gated on RFP $^{+}$cells). B-D, 293T cells were transfected with MSCV-GFP-RFP, MSCV-(SAP-1-GFP)-RFP, or MSCV-(SAP-2-GFP)-RFP. Levels of GFP fluorescence were used as a surrogate marker for SAP expression (B). Transfected cells were divided into 12 gates according to RFP levels (C) and the corresponding GFP level in each of the 12 gates was plotted in (D) for the three groups. Data represent two experiments.

moters drive the RFP and SAP-GFP fusion expression on the same construct, the RFP fluorescence indicates the level of transfection, while the GFP fluorescence directly reflects levels of SAP-1/-2 or GFP protein expression. When gated on $\mathrm{RFP}^{+}$transfected cells, the SAP-2-GFP group exhibited minimal levels of GFP fluorescence, as compared to either SAP-1-GFP or GFP group (Figure 5B). Quantitatively, when the level of GFP fluorescence was normalized against the level of RFP fluorescence, the SAP-2 group exhibited the lowest GFP/RFP ratio (Figure 5C and D). Collectively, these data indicate that SAP-2 is unstable, probably being rapidly degraded in the cell, and therefore non-functional.

We have serendipitously identified a new isoform of murine Sh2dla transcript that results from inclusion of a cryptic exon2' and encodes a 144-aa protein. Interestingly, whereas the cryptic exon $2^{\prime}$ is closer to exon- 1 than the exon 2 and it has classic intron acceptor and donor sites before and after it, respectively, overall SAP transcription favors the exclusion of exon2', and SAP-2 accounts for approximately $1 \%-3 \%$ of the total SAP transcription. It is possible that histone modifications at those intronic regions surrounding exon $2^{\prime}$ promote its preferential exclusion [24]. On the other hand, it is interesting to note that human SH2D1A gene has at least two alternatively spliced isoforms in addition to the 128-aa isoform 1 . One isoform is 3 amino acids shorter due to the use of an alternative in-frame splicing site within exon3 (NCBI accession: AAC79714.1), while the other isoform uses yet another different alternative splicing site within exon3, leading to a frame shift and truncated protein [7]. Therefore, in both human and mouse, SAP has multiple isoforms. Our results indicate that SAP-2 protein per se is non-functional and unstable. However, it is possible that SAP-2 transcription is modulated in particular cell populations and/or at particular stages of cell activation or differentiation. For example, $24 \mathrm{~h}$ following activation, CD8 $\mathrm{T}$ cells but not CD4 $\mathrm{T}$ cells possess increased expression of the SAP-2 mRNA, while the expression of total SAP and, by inference, the SAP-1 isoform is downregulated. It would be interesting to see in the future whether alternative splicing, in either human or mouse, is an operational mechanism by which SAP-mediated immune functions are regulated in a cell- and context-specific manner.

We thank Drs. Pamela Schwartzberg from National Institutes of Health and Andre Veillette from Clinical Research Institute of Montreal for kindly sharing reagents. This work was supported by funds from the Tsinghua-Peking Center for Life Sciences and the National Natural Science Foundation of China (81072464).

1 Purtilo DT, Cassel CK, Yang JP, Harper R. X-linked recessive progressive combined variable immunodeficiency (duncan's disease). Lancet, 1975, 1: 935-940

2 Engel P, Eck MJ, Terhorst C. The SAP and SLAM families in immune responses and $\mathrm{X}$-linked lymphoproliferative disease. Nat Rev Immunol, 2003, 3: 813-821 
3 Grierson HL, Skare J, Hawk J, Pauza M, Purtilo DT. Immunoglobulin class and subclass deficiencies prior to Epstein-Barr virus infection in males with $\mathrm{X}$-linked lymphoproliferative disease. Am J Med Genet, 1991, 40: 294-297

4 Skare JC, Sullivan JL, Milunsky A. Mapping the mutation causing the X-linked lymphoproliferative syndrome in relation to restriction fragment length polymorphisms on Xq. Hum Genet, 1989, 82: 349-353

5 Skare JC, Milunsky A, Byron KS, Sullivan JL. Mapping the X-linked lymphoproliferative syndrome. Proc Natl Acad Sci USA, 1987, 84: 2015-2018

6 Nichols KE, Harkin DP, Levitz S, Krainer M, Kolquist KA, Genovese C, Bernard A, Ferguson M, Zuo L, Snyder E, Buckler AJ, Wise C, Ashley J, Lovett M, Valentine MB, Look AT, Gerald W, Housman DE, Haber DA. Inactivating mutations in an SH2 domain-encoding gene in X-linked lymphoproliferative syndrome. Proc Natl Acad Sci USA, 1998, 95: 13765-13770

7 Sayos J, Wu C, Morra M, Wang N, Zhang X, Allen D, van Schaik S, Notarangelo L, Geha R, Roncarolo MG, Oettgen H, De Vries JE, Aversa G, Terhorst C. The X-linked lymphoproliferative-disease gene product SAP regulates signals induced through the co-receptor SLAM. Nature, 1998, 395: 462-469

8 Cannons JL, Tangye SG, Schwartzberg PL. SLAM family receptors and SAP adaptors in immunity. Annu Rev Immunol, 2010, 29: 665-705

9 Crotty S, Kersh EN, Cannons J, Schwartzberg PL, Ahmed R. SAP is required for generating long-term humoral immunity. Nature, 2003, 421: $282-287$

10 Qi H, Cannons JL, Klauschen F, Schwartzberg PL, Germain RN. SAP-controlled T-B cell interactions underlie germinal centre formation. Nature, 2008, 455: 764-769

11 Kageyama R, Cannons JL, Zhao F, Yusuf I, Lao C, Locci M, Schwartzberg PL, Crotty S. The receptor LY108 functions as a SAP adaptor-dependent on-off switch for T cell help to B cells and NKT cell development. Immunity, 2012, 36: 986-1002

12 Zhao F, Cannons JL, Dutta M, Griffiths GM, Schwartzberg PL. Positive and negative signaling through SLAM receptors regulate synapse organization and thresholds of cytolysis. Immunity, 2012, 36: 1003-1016

13 Coffey AJ, Brooksbank RA, Brandau O, Oohashi T, Howell GR, Bye JM, Cahn AP, Durham J, Heath P, Wray P, Pavitt R, Wilkinson J, Leversha M, Huckle E, Shaw-Smith CJ, Dunham A, Rhodes S, Schuster V, Porta G, Yin L, Serafini P, Sylla B, Zollo M, Franco B, Bolino A, Seri M, Lanyi A, Davis JR, Webster D, Harris A, Lenoir G, de St Basile G, Jones A, Behloradsky BH, Achatz H, Murken J, Fassler R, Sumegi J, Romeo G, Vaudin M, Ross MT, Meindl A, Bentley DR. Host response to EBV infection in X-linked lymphoproliferative disease results from mutations in an $\mathrm{SH}$ 2-domain encoding gene. Nat Genet, 1998, 20: 129-135

14 Czar MJ, Kersh EN, Mijares LA, Oohashi T, Howell GR, Bye JM, Cahn AP, Durham J, Heath P, Wray P, Pavitt R, Wilkinson J, Leversha M, Huckle E, Shaw-Smith CJ, Dunham A, Rhodes S, Schuster V, Porta G, Yin L, Serafini P, Sylla B, Zollo M, Franco B, Bolino A, Seri M, Lanyi A, Davis JR, Webster D, Harris A, Lenoir G, de St Basile G, Jones A, Behloradsky BH, Achatz H, Murken J, Fassler R, Sumegi J, Romeo G, Vaudin M, Ross MT, Meindl A, Bentley DR. Altered lymphocyte responses and cytokine production in mice deficient in the X-linked lymphoproliferative disease gene SH2D1A/DSHP/SAP. Proc Natl Acad Sci USA, 2001, 98: 7449-7454

15 Livak KJ, Schmittgen TD. Analysis of relative gene expression data using real-time quantitative PCR and the $2^{-\Delta \Delta C_{\mathrm{T}}}$ method. Methods, 2001, 25: 402-408

16 Xu H, Li X, Liu D, Li J, Zhang X, Chen X, Hou S, Peng L, Xu C, Liu W, Zhang L, Qi H. Follicular T-helper cell recruitment governed by bystander B cells and ICOS-driven motility. Nature, 2013, 496: 523-527

17 Veillette A, Zhang S, Shi X, Dong Z, Davidson D, Zhong MC. SAP expression in $\mathrm{T}$ cells, not in B cells, is required for humoral immunity. Proc Natl Acad Sci USA, 2008, 105: 1273-1278

18 Pandya-Jones A. Pre-mRNA splicing during transcription in the mammalian system. Wiley Interdiscip Rev RNA, 2011, 2: 700-717

19 Wu C, Nguyen KB, Pien GC, Wang N, Gullo C, Howie D, Sosa MR, Edwards MJ, Borrow P, Satoskar AR, Sharpe AH, Biron CA, Terhorst C. SAP controls $\mathrm{T}$ cell responses to virus and terminal differentiation of TH2 cells. Nat Immunol, 2001, 2: 410-414

20 Wu C, Sayos J, Wang N, Howie D, Coyle A, Terhorst C. Genomic organization and characterization of mouse SAP, the gene that is altered in X-linked lymphoproliferative disease. Immunogenetics, 2000, 51: 805-815

21 Latour S, Gish G, Helgason CD, Humphries RK, Pawson T, Veillette A. Regulation of SLAM-mediated signal transduction by SAP, the $\mathrm{X}$-linked lymphoproliferative gene product. Nat Immunol, 2001, 2: 681-690

22 Poy F, Yaffe MB, Sayos J, Saxena K, Morra M, Sumegi J, Cantley LC, Terhorst C, Eck MJ. Crystal structures of the XLP protein SAP reveal a class of $\mathrm{SH} 2$ domains with extended, phosphotyrosineindependent sequence recognition. Mol Cell, 1999, 4: 555-561

23 Morra M, Lu J, Poy F, Martin M, Sayos J, Calpe S, Gullo C, Howie D, Rietdijk S, Thompson A, Coyle AJ, Denny C, Yaffe MB, Engel P, Eck MJ, Terhorst C. Structural basis for the interaction of the free SH2 domain EAT-2 with SLAM receptors in hematopoietic cells. EMBO J, 2001, 20: 5840-5852

24 de Almeida SF, Carmo-Fonseca M. Design principles of interconnections between chromatin and pre-mRNA splicing. Trends Biochem Sci, 2012, 37: 248-253

Open Access This article is distributed under the terms of the Creative Commons Attribution License which permits any use, distribution, and reproduction in any medium, provided the original author(s) and source are credited. 